\title{
From East to West and Return: A Western Interpretation of Acupuncture
}

\author{
Emilio Minelli ${ }^{1}$, Bruna Francescucci ${ }^{2}$, Valentino Toschi ${ }^{1}$, Stefano lotti ${ }^{3,4^{*}}$, Emil Malucelli ${ }^{3}$, Jeanette AM Maier ${ }^{1}$ \\ ${ }^{1}$ Department of Biomedical Sciences and Clinics L. Sacco, University of Milan, Via G.B. Grassi 74, Milano, Italy \\ ${ }^{2}$ Hospitaller Order of St. John of God, Fatebenefratelli, Via Cavour, 2, Cernusco sul Naviglio, Italy \\ ${ }^{3}$ Department of Pharmacy and Biotechnology, University Alma Mater of Bologna, Via San Donato 19/2, Bologna, Italy \\ ${ }^{4}$ National Institute of Biostructures and Biosystems, 00136 Rome, Italy
}

*Corresponding author: Stefano lotti, Department of Pharmacy and Biotechnology, University Alma Mater of Bologna, Via San Donato $19 / 2$, Bologna, Italy, Tel: +39 051 2095413; Fax: +39 051 2095625; E-mail: stefano.iotti@unibo.it

Received date: February 20, 2017; Accepted date: March 14, 2017; Published date: March 17, 2017

Copyright: ( 2017 Minelli E, et al. This is an open-access article distributed under the terms of the Creative Commons Attribution License, which permits unrestricted use, distribution, and reproduction in any medium, provided the original author and source are credited.

\begin{abstract}
Acupuncture, a complementary therapy originated in China over 3000 years ago, is widely practiced in western countries. There is an urgent need to define the mechanisms underlying some clinical effects obtained by acupuncture. A placebo effect is sustained by neuroimaging evidence indicating the activation of areas in the brain that are involved in expectation after real and sham acupuncture. However, only real acupuncture modulates the activity of specific regions in the brain some of which involved in the perception of pain. Basic research applied to the field of acupuncture is beginning to offer a scientific interpretation to these events. Indeed, the stimulation of acupoints alters the architecture of the connective tissue and this local mechanical stress is delivered to the keratinocytes, mast cells and fibroblasts, which react in several manners, including the release of various molecules that act on peripheral nerve endings. Consequently, locally generated signals can be transmitted to the neural centers. In the light of these achievements and urging more research in the field, it can be concluded that there are common denominators between acupuncture and western medicine.
\end{abstract}

Keywords: Acupuncture; Complementary therapy

\section{Introduction}

The Jesuit missionaries, who introduced acupuncture in France in the 16th century, would be astonished by the spreading of this age-old healing practice in Europe and in the New World. Since 1997 the National Institute of Health has documented the efficacy of this form of alternative medicine in several conditions, primarily for its analgesic effects, so that acupuncture is now used in pain clinics and hospices world- wide [1,2]. Acupuncture seems also to improve sleep, digestive function, and in general, sense of well-being. More than 100 Cochrane reviews are available [3]. Only two clear evidence based indications emerge, i.e. acupuncture helps in case of nausea/vomit and in chronic or episodic tension-type headaches. In the treatment of chronic low back pain, real or sham (nonspecific, placebo) acupuncture demonstrates higher rates of improvement than with conventional therapies [4]. Back in 1998, Dr. Marcia Angell, former editor-in-chief of the New England Journal of Medicine, wrote that "there cannot be two kinds of medicine-conventional and alternative. There is only medicine that has been adequately tested and medicine that has not" [5], thus urging to search for the biologic mechanisms of any therapeutic approach. Some efforts have been made to understand how acupuncture works in terms of the principles of western medical biochemistry and physiology [6,7]. In addition, the study of acupuncture has offered new insights into biomedicine [8].

\section{The Cultural Context Counts}

All the cultures developed their own philosophy of science and, therefore, their own medicine. Western medicine originates from Hippocrates, who abandoned the archaic interpretation of a disease as something supernatural to introduce the concept that a disease results from imbalances caused by environmental factors, life-style and diet. More or less at the same time in China, acupuncture, one of the facets of Traditional Chinese Medicine (TCM), emerged with the aim of preventing diseases and, eventually, curing them [9]. According to Taoism, health results from the balance between yin and yang, which allows the harmonious flow of vital energy, the so-called Qi, through conduits defined meridians. Disruption and blocks of Qi's flow generate illness. By inserting thin needles into strategic points along these meridians, acupuncture restores the correct flow of energy, thus activating healing. The semantics of TCM sounds archaic and very distant from the current medical language developed in western countries. Interestingly, western interpretation of disease as an alteration of homeostasis is not so different from the yin/yang imbalance described in ancient Chinese texts and also recalls the Hippocratic concept of imbalances of the four humors. Moreover, since Man is an integral part of his surroundings, ancient Chinese practitioners evaluated any individual within his context in the Universe. Indeed, internal energies reflect the elemental forces of the universe, thus underscoring that social, climatic, nutritional and seasonal factors influence health. These topics recall the definition of health provided by the World Health Organization (WHO) as a complete physical, mental and social well-being. Also western clinicians collect information about nutrition, life style and psychological factors to help the diagnostic process and improve the treatment.

Here we summarize a couple of examples showing that, in spite of the profound differences in the approach to diagnose and treat diseases, acupuncture and conventional medicine are less distant than previously thought. 
Let us look at menopause with the eyes of an acupuncturist or a western clinician. It is relevant that menopause is viewed as a physiological process by both the perspectives, but is described with different words. From TCM viewpoint, menopause is GENG NIAN QI, which can be translated as means age of deep changes, and is due to a deficiency of the "Kidney Qi", leading to an imbalance between yin and yang, which explains endocrine, somatic and psychic changes typical of menopause. The acupuncturist will therefore choose acupoints that nourish the "kidney system" which, according to Chinese medicine, stores energy, governs bones, marrow and brain. Moreover, the "kidney" dominates water metabolism, and associates with "cold". Therefore, working on the "kidney system" helps to counterbalance symptoms typically associated with menopause, such as hot flashes, sweats, palpitations, osteoporosis and depression [10]. Conventional western medicine describes menopause as the results from the reduced production of estrogen with consequent endocrine imbalance, which causes the aforementioned disorders. To relieve symptoms and signs, the western clinician might utilize hormone replacement therapy or eventually prescribe antidepressants or medications to prevent or treat osteoporosis. The two approaches are different, but the final aim is to generate a novel balance in a time of deep changes for women.

Another interesting instance comes from studies on rheumatoid arthritis (RA). On the basis of the diagnostic process, the acupuncturist divides patients with RA in "heat" and "cold" groups and treats them differently. Wietmarschen et al. phenotyped RA patients using metabolomics, proteomics and biochemical signature and found significant differences between patients with RA defined "heat" or "cold" $[11,12]$. In this case, TCM diagnosis allowed to identify markers to characterize different subgroups of patients with the obvious aim of tailoring therapies to improve outcomes.

\section{Do Acupoints Exist?}

At the moment, no definitive scientific evidence about the existence of acupoints is available. Although several studies on this topic are available, major limitations -such as lack of statistical analysis and small sample size-jeopardize their significance and interpretation.

Many Western practitioners consider the acupuncture points as trigger points $[8,13]$. This is one topic where western medicine and acupuncture converge, since trans-cutaneous electrical nerve stimulation (TENS), largely used for pain relief, was developed on these bases [8].

Some acupoints correspond to spots with high density of neurovascular bundles [14]. On the other hand, in a pilot study performed on nine autopsy cases, immunohistochemistry on skin biopsies showed a decreased number and density of subcutaneous nerves in acupoints [15].

Several authors insist on the unique electrical properties of acupoints $[14,16]$. In a study on 28 healthy adults, a statistically significant reduction of electrical impedance was observed in acupoints of the Large Intestine meridian. Interestingly, these acupoints associated with increased ultrasound echogenicity due to the presence of collagen [17]. It is noteworthy that $80 \%$ of acupoints are located on fascial planes and this means that the insertion and manipulation of the needle generate more tissue stress than in other parts [18]. Even though low electrical impedance and associated collagenous bands represent the most compelling evidence available at the moment, there is no conclusive indication about the fact that acupoints show electrically distinguishable characteristics. On the other hand, skin electric impedance depends on the thickness of the skin, the integrity of the stratum corneum, and the degree of hydration, which is known to affect the lipid composition of the membranes as well as the dynamics of the keratin filament terminals [19]. Also the size and the shape of the electrodes and other technical cues might complicate the interpretation of the results [20].

In spite of these inconclusive evidences, it should be recalled that modern imaging techniques, such as functional Magnetic Resonance Imaging (fMRI) and positron emission tomography (PET), indicate that acupuncture modulates brain activity. Considering the wide spread use of acupuncture in the control of pain, it is of relevance that needle stimulation of particular acupoints increases the signals in the region of the brain involved in pain perception as detected by fMRI [21]. However, the mechanisms underlying acupuncture-induced analgesia remain elusive.

\section{What is Known about the Mechanisms of Action of Acupuncture?}

Translating the theory of acupuncture into a western paradigm is rather complex. However, the identification of the mechanisms of action of acupuncture is important to foster its integration with conventional medicine. Research in animal and ex vivo models have shown that acupuncture stimulates the release of various molecules, probably via mechanotransduction, the complex process through which the cell translates mechanical stimuli in biochemical signals that modulate cell response.

Initially, it was shown that the insertion and manipulation of needles in acupoints determined a biomechanical response characterized by alterations of the architecture of the connective tissue in animal models as well as in humans [22]. Then, the same authors elegantly demonstrated in mice that acupuncture induces an active cytoskeletal reorganization of fibroblasts up to several centimeters away from the needle [23]. These results are intriguing, since the cytoskeleton acts as a mechanotransducer thus modulating cell functions, regulates the homeostasis of the extracellular matrix [24] and the secretion of various molecules [25]. Regarding the effects on extracellular matrix, it should be recalled that acupuncture and other physical-based therapies have been shown to reduce tissue stiffness and connective tissue inflammation and this might help in several pathological conditions [26]. Under a western point of view, it is undeniable that these studies have broadened our knowledge about connective tissue and mechanotransduction.

Compelling evidence about the local effects of acupuncture derives from the study by Park et al. who demonstrate the activation of ERK in the keratinocytes of the epidermis and in the fibroblasts of the dermis in the isolated skin layer of mice 1 hour after acupuncture [27]. It is noteworthy that ERK, which is rapidly activated by mechanical stimuli, regulates many crucial cellular functions. Another biomechanical response to the insertion of needles in some acupoints in rats is the degranulation of mast cells, which are rather abundant at the site of acupoints [28]. Interestingly, not only acupuncture but also other physical cues such as laser light and heat stimulate mast cell degranulation, probably through the activation of TRPV2 [29], a member of transient receptor potential ion channels, which is permeable to calcium. The mediators released by mast cells promote extracellular matrix remodeling and stimulate nerve endings [30], events that might mediate, in part, the increase of pain threshold. A particular attention has been devoted to the local release of purines by 
keratinocytes, fibroblasts and mast cells after acupuncture. Extracellular ATP acts as a neurotransmitter by binding the purinergic receptors, which are abundant in the central and peripheral nervous systems [31], and is rapidly catabolized by a cascade of ectoenzymes to adenosine. Adenosine is a crucial mediator that regulates tissue function by activating four different types of adenosine receptors (A1, A2A, A2B and A3) [32]. In a murine model, Goldman et al. showed that adenosine markedly increases during and after acupuncture and exerts its anti-nociceptive effects by activating the A1 receptor on the ascending nerves to deliver locally generated signals to neural centers [33]. Part of the beneficial effect of adenosine might also depend on its anti-inflammatory activity, mainly mediated by its binding to the A2 receptors [32]. It is noteworthy that the interstitial concentration of adenosine is significantly increased during and after acupuncture also in humans [34]. On these bases, it can be hypothesized that adenosine stimulates sensory nerve endings in the skin and sends impulses which modulate the activities of the neurons in the brain stem and hypothalamus to inhibit signaling to the pain centers in the cortex and also to shape autonomic functions.
Several studies suggest that acupuncture triggers the release of the endogenous opioids $\beta$-endorphin and enkefalin, which produce analgesia by binding to the $\mu$-subtype opioid receptors [35]. In the peripheral nervous system, $\beta$-endorphin inhibits the release of substance $\mathrm{P}$, a potent algogenic peptide. In the central nervous system, endogenous opioids inhibit the release of GABA, leading to increased production of dopamine, which reduces pain perception. In chronic pain patients diagnosed with fibromyalgia, PET showed that acupuncture evokes a short- and long-term increase in $\mu$-opioid receptor availability in areas involved in pain processing, such as the caudate, thalamus, cingulate, insula, and amygdala, and this correlates with better clinical outcomes [36]. These findings highlight the involvement of endogenous opioids and their receptors in mediating some effects of acupuncture. Figure 1 summarizes present knowledge about the mechanisms of action of acupuncture.
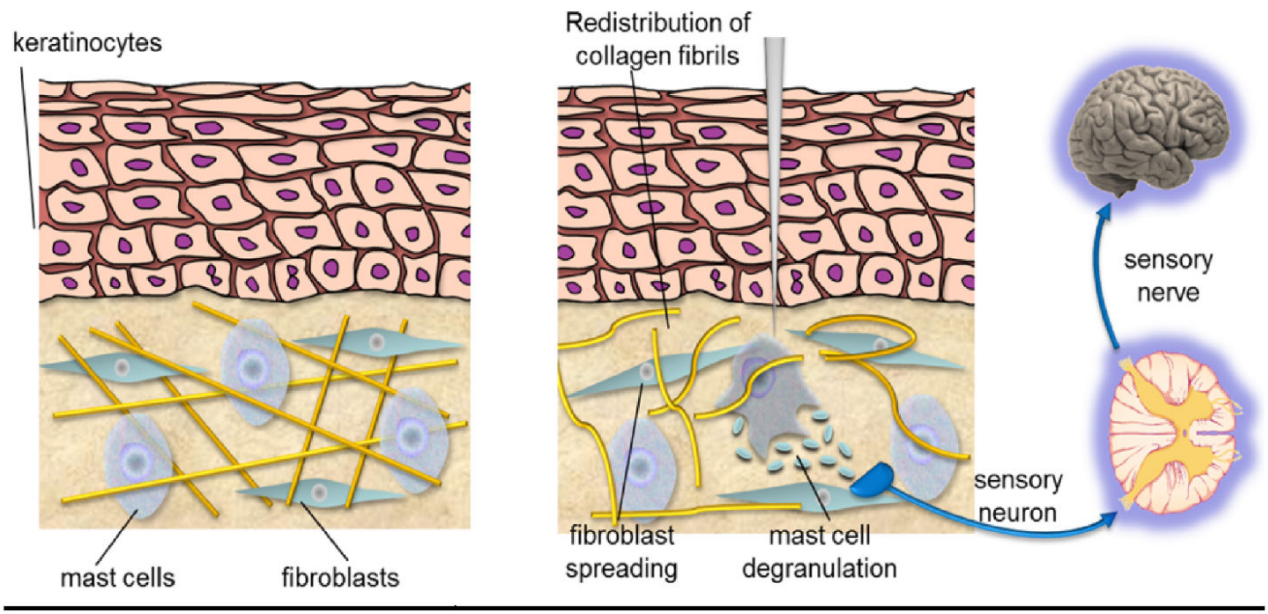

Figure 1: A summary of the potential mechanisms of action of acupuncture. (After stimulating an acupoint with a needle, the architecture of the connective tissue is altered. Collagen fibers are reorganized, fibroblasts spread, and mast cells degranulate. Mediators released by the cells stimulate nerve endings with consequent activation of different areas in the central nervous system).

\section{Can East and West Meet?}

It should be underscored that several studies on the mechanisms of action of acupuncture were performed in experimental models, and it is not easy to transduce the results obtained in animals to humans, partly because the psychosocial context impacts on the outcome of acupuncture. Accordingly, while only real acupuncture has specific effects on the brain as demonstrated by neuroimaging, both real and sham acupuncture activate areas in the central nervous system that are involved in expectation and belief [37]. These results from acupuncture research shed some light into our understanding of the placebo effect in clinical care. In general, all the studies performed to understand the analgesic effect of acupuncture have added new dowels into the complex mosaic of the physiology of pain.

\section{Conclusion}

More resources should be invested in basic and clinical research in the field of acupuncture. The development of graphene-modified acupuncture needles might represent the next-generation approach. These needles are biosensors that might be used to measure in vivo the molecular events triggered by acupuncture [38]. This approach links nanotechnology, electrochemistry and TCM, i.e. one more step towards the integration of TCM and western medicine.

\section{References}

1. National Institutes of Health (1998) Consensus Conference. Acupuncture. JAMA 280: 1518-1524. 
2. Wilkinson J, Faleiro JR (2007) Acupuncture in pain management. Contin Educ Anaesth Crit Care Pain 7: 135-138.

3. http://www.cochrane.org/search/site/acupuncture

4. Wang SM, Kain ZN, White PF (2008) Acupuncture analgesia: II. Clinical considerations. Anesth Analg 106: 611-621.

5. Angell M, Cassirer JP (1998) Alternative Medicine- the risks of untested and unregulated remedies. New Engl J Med 339: 839-841.

6. Wang SM, Kain ZN, White P (2008) Acupuncture analgesia: I. The scientific basis. Anesth Analg 106: 602-610.

7. Kaptchuk TJ (2002) Acupuncture: theory, efficacy, practice. Ann Intern Med 136: 374-383.

8. MacPherson H, Hammerschlag R, Coeytaux RR, Davis RT, Harris RE, et al. (2016) Unanticipated Insights into Biomedicine from the Study of Acupuncture. J Altern Complement Med. 22: 101-107.

9. White A, Ernst E (2004) A brief history of acupuncture. Rheumatol. 43: 662-663.

10. Avis NE, Coeytaux RR, Isom S, Prevette K, Morgan T (2016) Acupuncture in Menopause (AIM) study: a pragmatic, randomized controlled trial. Menopause 23: 626-637.

11. van Wietmarschen H, Yuan K, Lu C, Gao P, Wang J, et al. (2009) Systems biology guided by Chinese medicine reveals new markers for sub-typing rheumatoid arthritis patients. J Clin Rheumatol 15: 330-337.

12. van Wietmarschen HA, Dai W, van der Kooij AJ, Reijmers TH, Schroën Y, et al. (2012) Characterization of rheumatoid arthritis subtypes using symptom profiles, clinical chemistry and metabolomics measurements. PLoS One 7: e44331.

13. Melzack R, Stillwell DM, Fox EJ (1977) Trigger points and acupuncture points for pain: correlations and implications. Pain 3: 3-23.

14. Juan L, Qing W, Huiling L, Haoxu D, Yan L, et al. (2012) Biophysical Characteristics of Meridians and Acupoints: A Systematic Review. Evid Based Complement Alternat Med Article ID 793841.

15. Wick F, Wick N, Wick MC (2007) Morphological analysis of human acupuncture points through immunohistochemistry. Am J Phys Med Rehabil 86: 7-11.

16. Ahn C, Wu J, Badger GJ, Hammerschlag R, Langevin HM (2005) Electrical impedance along connective tissue planes associated with acupuncture meridians. BMC Complement Altern Med 5: 10.

17. Ahn AC, Park M, Shaw JR, McManus CA, Kaptchuk TJ, et al. (2010) Electrical impedance of acupuncture meridians: the relevance of subcutaneous collagenous bands. PLoS One 5: e11907.

18. Langevin HM, Churchill DL, Cipolla MJ (2001) Mechanical signaling through connective tissue: A mechanism for the therapeutic effect of acupuncture. Faseb J 15: 2275-2282.

19. Björklund S, Ruzgas T, Nowacka A, Dahi I, Topgaard D, et al. (2013) Skin membrane electrical impedance properties under the influence of a varying water gradient. Biophys J 104: 2639-2650.

20. Ahn AC, Colbert AP, Anderson BJ, Martinsen OG, Hammerschlag R, et al. (2008) Electrical properties of acupuncture points and meridians: a systematic review. Bioelectromagnetics 29: 245-256.

21. Egorova N, Gollub RL, Kong J (2015) Repeated verum but not placebo acupuncture normalizes connectivity in brain regions dysregulated in chronic pain. NeuroImage: Clinical 9: 430-435.
22. Langevin HM, Konofagou EE, Badger GJ, Churchill DL, Fox JR, et al. (2004) Tissue displacements during acupuncture using ultrasound elastography techniques. Ultrasound Med Biol 30: 1173-1183.

23. Langevin HM, Bouffard NA, Badger GJ, Churchill DL, Howe AK. (2006) Subcutaneous tissue fibroblast cytoskeletal remodeling induced by acupuncture: evidence for a mechanotransduction-based mechanism. J Cell Physiol 207: 767-774.

24. Humphrey JD, Dufresne ER, Schwartz MA (2014) Mechanotransduction and extracellular matrix homeostasis. Nat Rev Mol Cell Biol 15: 802-812.

25. Bouffard NA, Cutroneo KR, Badger GJ, White SL, Buttolph TR, et al. (2008) Tissue stretch decreases soluble TGF-betal and type-1 procollagen in mouse subcutaneous connective tissue: evidence from ex vivo and in vivo models. J Cell Physiol 214: 389-395.

26. Langevin HM, Keely P, Mao J, Hodge LM, Schleip R (2016) Connecting (T)issues: How Research in Fascia Biology Can Impact Integrative Oncology. Cancer Res. 76: 6159-6162.

27. Park JY, Park JJ, Jeon S, Doo AR, Kim SN, et al. (2014) From peripheral to central: the role of ERK signaling pathway in acupuncture analgesia. J Pain 15: 535-549.

28. Ding GH, Zhang D, Huang M, Wang LN, Yao W, et al. (2013) Function of Collagen and Mast Cells in Acupuncture Points. Current research in acupuncture. (1st edn) Springer. New York.

29. Zhang D, Spielmann A, Wang L, Ding G, Huang F, et al. (2012) Mast-cell degranulation induced by physical stimuli involves the activation of transient-receptor-potential channel TRPV2. Physiol Res. 61: 113-124.

30. Wernersson S, Pejler G (2014) Mast cell secretory granules: armed for battle. Nat Rev Immunol 14: 478-494.

31. Abbracchio MP, Burnstock G, Verkhratsky A, Zimmermann H (2009) Purinergic signalling in the nervous system: an overview. Trends Neurosci 32: 19-29.

32. Haskó G, Linden J, Cronstein B, Pacher P (2008) Adenosine receptors: therapeutic aspects for inflammatory and immune diseases. Nat Rev Drug Discov 7: 759-770.

33. Goldman N, Chen M, Fujita T, Xu Q, Peng W, et al. (2010) Adenosine Al receptors mediate local anti-nociceptive effects of acupuncture. Nat Neurosci 13: 883-888.

34. Takano T, Chen X, Luo F, Fujita T, Ren Z, et al. (2012) Traditional acupuncture triggers a local increase in adenosine in human subjects. J Pain 13: 1215-1223.

35. Han JS (2004) Acupuncture and endorphins. Neurosci Lett 361: 258-261.

36. Harris RE, Zubieta JK, Scott DJ, Napadow V, Gracely RH, et al. (2009) Traditional Chinese acupuncture and placebo (sham) acupuncture are differentiated by their effects on mu-opioid receptors (MORs). Neuroimage 47: 1077-1085.

37. Pariente J, White P, Frackowiak RS, Lewith G (2005) Expectancy and belief modulate the neuronal substrates of pain treated by acupuncture. Neuroimage 25: 1161-1167.

38. Tang L, Du D, Yang F, Liang Z, Ning Y, et al. (2015) Preparation of Graphene-Modified Acupuncture Needle and Its Application in Detecting Neurotransmitters. Sci Rep 5: 11627. 